\title{
Hypertension Cure and Reducing Pill Burden after Adrenalectomy for Endocrine Hypertension of Adrenal Origin: A Comparative Study from an Asian and UK Cohort
}

\author{
Ciaran Durand ${ }^{1}$, Titus C Vasciuc ${ }^{2}$, Chia H Tai $^{3}$, Raluca Orpean ${ }^{4}$, Fiona Eatock ${ }^{5}$, Mehak Mahipal ${ }^{6}$, Tan W Boon ${ }^{7}$, Kee Y Ngiam $^{8}$, \\ Rajeev Parameswaran ${ }^{9}$
}

\begin{abstract}
Background: The cure rate of endocrine hypertension following adrenalectomy is heterogeneous. Our aim of the study was to investigate the etiology and cure rate of endocrine hypertension between an Asian and UK cohort.

Materials and methods: This is a retrospective study of patients who underwent adrenalectomy from two tertiary centers in Singapore (cohort I) and the UK (cohort II) for endocrine hypertension. Demographic, clinical details, blood pressure data, and cure rates of hypertension were collected and analyzed.

Results: Cohort I included 115 patients (59F:56M), the mean age of 50.05 (SD 12.82), and cohort II had 128 patients (65F:63M), the mean age of 52.88 (SD 14.45) during the study period. There was a higher incidence of Conn's adenoma in cohort l, with predominantly left-sided tumors and grade I and II hypertension at presentation $(p=0.001)$. In contrast, cohort II had pheochromocytoma (PCC) as the most frequent diagnosis, right-sided tumors, and grade II and III hypertension $(p=0.001)$. Significant differences in the mean pre-op systolic and diastolic BP and choice of antihypertensive medications between the two cohorts were noted $(p=0.001)$. The hypertension cure rates were similar $(70$ vs $69 \%)$ between the two cohorts. In patients where no cure was achieved, adrenalectomy resulted in a decrease in the pill burden (decreased number of classes and standard dose) in both the cohorts $(p=0.03$ ). The complications rates and overall mortality were comparable between the cohorts.

Conclusion: The etiology of adrenal hypertension is different between the East and the West. Adrenalectomy cured hypertension in the majority of the patient and reduced pill burden where no cure for hypertension was achieved.

Keywords: Adrenal, Adrenalectomy, Aldosterone-producing adenoma, Cushing's adenoma, Hypertension, Pheochromocytoma.

World Journal of Endocrine Surgery (2021): 10.5005/jp-journals-10002-1314
\end{abstract}

\section{InTRODUCTION}

Arterial hypertension is the condition where there is a nonphysiological persistent elevation of systemic blood pressure defined as a resting systolic BP (SBP) $\geq 140 \mathrm{~mm} \mathrm{Hg}$ or diastolic BP (DBP) $\geq 90 \mathrm{~mm} \mathrm{Hg}$. The condition is a major cardiovascular risk factor for stroke, ischemic heart disease, and heart failure. ${ }^{1}$ It is estimated that roughly $10 \%$ of the global population has essential hypertension which has no identifiable cause. The prevalence of hypertension for adults aged between 30 years and 69 years was $26 \%$ for men and $21 \%$ for women in Singapore, compared with $31 \%$ among men and $26 \%$ among women in the UK, based on National Health Statistics. On the other hand, the prevalence of secondary hypertension was thought to be around $5 \%$ but more recent studies have shown the prevalence to be closer to $20 \%,{ }^{2}$ especially in younger patients with hypertension. ${ }^{3}$ The most common causes of secondary hypertension are renovascular disease and endocrine conditions. $^{4}$

Presentation with hypertension is common in many endocrine conditions due to hormonal imbalances associated with the gland of origin. Three endocrine conditions commonly presenting with hypertension include primary aldosteronism (PA), conditions causing glucocorticoid excess (Cushing's syndrome), and pheochromocytoma-paraganglioma syndromes (PCC/PGL). Adrenalectomy is the standard treatment for patients with
${ }^{1}$ Department of Endocrine Surgery, Queens University Belfast, Belfast, Northern Ireland, United Kingdom

${ }^{2}$ Department of Endocrine Surgery, Leeds Teaching Hospitals, Leeds, United Kingdom

3,6,9 Department of Endocrine Surgery, National University Hospital, Singapore

${ }^{4}$ Department of Endocrinology, Belfast Health and Social Care Trust, Belfast, Northern Ireland, United Kingdom

${ }^{5}$ Department of Endocrine Surgery, Belfast Health and Social Care Trust, Belfast, Northern Ireland, United Kingdom

${ }^{7}$ Department of General Surgery, National University Hospital, Singapore

${ }^{8}$ Department of Surgery, National University Hospital, Singapore

Corresponding Author: Rajeev Parameswaran, Department of Endocrine Surgery, National University Hospital, Singapore, Phone: +65 91271524, e-mail: rajeev_parameswaran@nuhs. edu.sg

How to cite this article: Durand C, Vasciuc TC, Tai CH, et al. Hypertension Cure and Reducing Pill Burden after Adrenalectomy for Endocrine Hypertension of Adrenal Origin: A Comparative Study from an Asian and UK Cohort. World J Endoc Surg 2021;13(1):9-15.

Source of support: Nil

Conflict of interest: None

(o) The Author(s). 2021 Open Access This article is distributed under the terms of the Creative Commons Attribution 4.0International License (https://creativecommons. org/licenses/by-nc/4.0/), which permits unrestricted use, distribution, and non-commercial reproduction in any medium, provided you give appropriate credit to the original author(s) and the source, provide a link to the Creative Commons license, and indicate if changes were made. The Creative Commons Public Domain Dedication waiver (http://creativecommons.org/publicdomain/zero/1.0/) applies to the data made available in this article, unless otherwise stated. 
Hypertension Cure and Reducing Pill Burden after Adrenalectomy for Endocrine Hypertension of Adrenal Origin

endocrine hypertension and offers the patient a realistic chance of cure..$^{5-8}$ It is not uncommon to see residual hypertension in patients treated for endocrine hypertension, due to the fact that some component of essential hypertension exists in these patients, especially in the elderly.

The prevalence of PA is estimated to be about $30 \%$ in hypertensive patients. ${ }^{9}$ The prevalence of PA increases with the grade of hypertension, seen in about $2 \%$ of those with grade I hypertension and up to $20 \%$ of patients with resistant hypertension. ${ }^{9,10}$ Similarly, in patients with endogenous Cushing's syndrome, hypertension is seen in up to $80 \%$ of adults and $60 \%$ of children but maybe even as high as $95 \%$ in ectopic ACTH secretion..$^{11-13}$ The third adrenal condition causing hypertension is a PCC-PGL, wherein patients may have paroxysmal hypertension but a third present with persistent hypertension. ${ }^{14}$ The response of hypertension to adrenal surgery varies with the underlying condition and other parameters such as gender and duration of hypertension. ${ }^{15,16}$ It should be mentioned that adrenalectomy for Cushing's syndrome is not to cure hypertension through a good proportion of patients present with hypertension, unlike PA and PCC/PPGL.

We hypothesize that the incidence of adrenal conditions causing hypertension varies with geography and therefore the cure rate of adrenalectomy to treat hypertension should differ as well. Our study aimed to compare the outcomes of cure following adrenalectomy for endocrine hypertension between an Asian and UK cohort.

\section{Materials and Methods}

The study is a retrospective analysis of patients who underwent adrenalectomy for endocrine hypertension in two tertiary centers, one from the East (cohort I: National University Hospital, Singapore) and the West (cohort II: Royal Victoria Hospital, Belfast). The study period for the cohort from Singapore was between 2001 and $2018(n=128)$ and for the UK cohort the study period was 2009 to 2018 ( $n=135$ ). Data for the period 2001 to 2008 in the UK cohort were very limited and hence not used in the analysis. The demographic details collected were age, gender, race, and clinicopathological parameters collected were diagnosis, nature of surgery (laparoscopic vs open), grade of hypertension, class, and a number of pre- and postoperative blood pressure medications, the cure rate of hypertension and mortality. Exclusion criteria were patients who were normotensive at presentation (13 from cohort I and 7 from cohort II). Institutional Board Review approval was obtained for the study from both the centers.

\section{Diagnosis of the Endocrine Conditions}

Standard diagnostic tests were used at both centers to confirm the diagnosis of the underlying adrenal condition. For PA, saline loading tests were performed to confirm autonomous aldosterone secretion in those with elevated plasma aldosterone levels or with elevated plasma aldosterone/plasma renin activity (PRA) ratio $(A R R)>50 \mathrm{ng} / 100 \mathrm{~mL} / \mathrm{ng} / \mathrm{mL} / \mathrm{h}$ with suppressed PRA values $<0.7 \mathrm{ng} / \mathrm{mL} / \mathrm{h}$. All PA patients underwent lateralization studies with adrenal vein sampling before adrenalectomy. The diagnosis of adrenal Cushing's was made following a non-suppressible cortisol level with $1 \mathrm{mg}$ overnight dexamethasone suppression test, along with elevated 24-hour urinary cortisol levels and a suppressed ACTH. Elevation of 24-hour urinary metanephrines performed on two separate occasions along with localization scans with computed tomography (CT) or magnetic resonance imaging (MRI) was used for diagnosis of PCC/PGL. In patients with PCC/PGL where there was a suspicion of metastasis I ${ }^{123}$ MIBG scan was used in the UK and DOTA-PET (due to non-availability of $\mathrm{I}^{123}$ ) in Singapore.

\section{Définitions}

As the definition of the grade of hypertension, we used the most current Guidelines for the management of Hypertension of the European Society of Cardiology and the European Society of Hypertension (ESC/ESH Task Force for the Management of Arterial Hypertension). ${ }^{17}$ Elevated BP (blood pressure) is considered as an office SBP at least $140 \mathrm{~mm} \mathrm{Hg}$ and/or DBP at least $90 \mathrm{~mm} \mathrm{Hg}$, which is equivalent to a $24-\mathrm{h}$ average blood pressure monitoring (ABPM) average of at least $130 / 80 \mathrm{~mm} \mathrm{Hg}$, or a home blood pressure monitoring (HBPM) average at least 135/85 mm Hg.(23).

The cure was classified as normalization of blood pressure after surgical therapy, and normalization of biochemical parameters, which included serum potassium and ARR, serum cortisol, and 24-hour urinary metanephrines for PCC/PPGL. Complete cure is defined as the state where the SBP and/or DBP normalized without any antihypertensive medications for a period of 1 year; Partial cure-where patients were normotensive following surgery and there was a reduction in the number of classes of antihypertensives or reductions in the dose of the antihypertensives. All patients were followed for a minimum of a period of 2 years following surgery.

\section{Classes of Antihypertensives}

The preferred drugs used in the treatment of hypertension include calcium channel blockers (CCB), beta-blockers (BB), angiotensinconverting enzyme inhibitors (ACEI), diuretics, and alpha-blockers $\left(A B^{\prime} s\right)$. The various drugs under each category and the standard doses used in the treatment of hypertension are shown in Table 1. The nature of the antihypertensives used depends on the underlying etiology of endocrine hypertension.

\section{Surgery}

All patients underwent surgery under general anesthesia after optimization of their blood pressures with antihypertensives described above. Most patients underwent laparoscopic surgery (anterior or posterior approach) for benign disease and those that were suspicious for malignant or deemed as large size unsuitable for laparoscopic surgery underwent open adrenalectomy.

\section{Statistical Analysis}

Categorical data are shown as rates and proportions, compared with the $X^{2}$ test, whereas continuous data were evaluated using the $t$-test, and the rest as means ( \pm standard deviation). Analysis of variance was used to compare the variables among the two cohorts. Changes in paired values (data before and after treatment) were analyzed using the paired $t$-test. Factors found to be significant on univariate analysis for cure were evaluated by multivariate analysis using the Cox proportional hazards regression model. $p$ values $<0.05$ were considered to be statistically significant.

\section{Results}

There were 115 patients (59F:56M) in cohort I and 128 patients (65F:63M) in cohort II during the study period as shown in Table 2. The mean age was comparable in the two cohorts [cohort I vs II: 50.05 (SD; 12.82) vs 52.88 (SD; 14.45)]. There was a significant difference in the race of patients recruited in the two centers whereby all patients were Caucasians from cohort II while in cohort I the Chinese ethnicity prevailed $(74 \%)(p=0.001)$. 
Table 1: Standard doses and the classes of antihypertensives used in the treatment of endocrine hypertension

\begin{tabular}{llll}
\hline $\begin{array}{l}\text { Calcium channel } \\
\text { blockers }\end{array}$ & $m g$ & Beta-blockers & $\mathrm{mg}$ \\
\hline Amlodipine (norvasc) & 5 & Atenolol & 50 \\
Nifedipine (adalat) & 30 & Bisoprolol & 5 \\
Verapamil & 180 & Carvedilol & 25 \\
Diltiazem & 180 & Metoprolol tartrate (IMM) & 100 \\
Felodipine (Plendil) & 5 & Propanolol & 80 \\
& & Labetalol & 200 \\
AR antagonists & $\mathrm{mg}$ & Timolol & 10 \\
Losartan & 50 & & \\
Valsartan & 80 & Vasodilators, alphaB & \\
Irbesartan & 150 & Prazosin & 1 \\
Candesartan & 8 & Terazosin & 1 \\
Telmisartan & 40 & Phenoxybenzamine & 20 \\
& & Hydralazine & 100 \\
ACE Inhibitors & $\mathrm{mg}$ & & \\
Captopril & 12.5 & Diuretics & $\mathrm{mg}$ \\
Enalapril & 5 & Frusemide (lasix) & 40 \\
Lisinopril & 10 & Spironolactone (aldactone) & 25 \\
Quinapril & 10 & Hydrochlorothiazide & 25 \\
Fosinopril & 40 & Amiloride & 5 \\
Cilazapril & 2.5 & Indapamide (thiazide) & 1.25 \\
Benazepril & 10 & Bumetanide (burinex) & 0.5 \\
Perindopril & 2 & Metolazone & 2.5 \\
& & Torsemide & 20 \\
\hline
\end{tabular}

The causes of endocrine hypertension were significantly different in the two cohorts $(p=0.001)$. In cohort I, surgery was performed for Conn's (67\%) followed by PCC/PGL (19\%) and Cushing's syndrome (14\%). In cohort II, most operations were performed for PCC/PGL (67\%), Conn's (19\%), and Cushing's syndrome (14\%). The majority of the cases in both the cohorts were performed laparoscopically ( 87 vs $82 \%$ ), using an anterior or posterior approach. Open procedures were slightly higher in cohort II (15 vs $10 \%$ in cohort I) and conversion rates were similar in both the cohorts (I vs II: 2.6 vs 3.1\%). Pathology was most common on the left side in cohort I $(69 / 115 ; 60 \%)$ whereas more common on the right side in cohort II $(69 / 128 ; 54 \%)(p=0.001)$. Bilateral surgery was more commonly observed in the cohort Il compared to cohort I (17 vs $5 \%)$.

In terms of hypertension, patients from cohort I had grade I and II hypertension whereas cohort II had more grade II and III hypertensions ( $p=0.001$ ) preoperatively. Following surgery, both cohorts had a similar response in their hypertension control with the majority in the normal or grade I blood pressure range (95 vs 92\%: $p=N S$ ). The mean pre-op and postoperative SBP and DBP were different in the two cohorts $(p=0.001)$ and are shown in Table 3 and Figure 1. In cohort II, the most commonly used class of drug was alpha-blockers whereas in cohort I calcium channel blocker was the most commonly used class of drug, and this correlates with the predominant pathology being treated at each center.

The overall cure of hypertension was similar in the two cohorts (SG vs UK: 70 vs $69 \%)(p=0.77)$ and there was no significant difference in cure rate between the various pathologies [Conn's (74:63\%) $(p=0.30)$ vs PCC/PPGL (73:69\%) $(p=0.46)$ and Cushing's $(50: 78 \%)(p=0.09)]$ treated. There was no significant change in the

Table 2: Clinicopathological parameters of the two cohorts

\begin{tabular}{|c|c|c|c|c|}
\hline Parameters & & Cohort I cohort (East) $(n=115)$ & Cohort II cohort (West) $(n=128)$ & $p$ value \\
\hline Age & Mean (SD) & $50.05(12.82)$ & $52.88(14.45)$ & 0.28 \\
\hline \multirow[t]{2}{*}{ Gender } & Female & 59 & 65 & 0.93 \\
\hline & Male & 56 & 63 & \\
\hline \multirow[t]{4}{*}{ Race } & Chinese & 85 & 0 & 0.001 \\
\hline & Malay & 12 & 0 & \\
\hline & India & 3 & 0 & \\
\hline & Caucasian & 15 & 128 & \\
\hline \multirow[t]{3}{*}{ Diagnosis } & Conn's & 77 & 24 & 0.001 \\
\hline & Cushing's & 16 & 18 & \\
\hline & PCC & 22 & 86 & \\
\hline \multirow[t]{3}{*}{ Surgery } & Laparoscopic & 100 & 105 & 0.35 \\
\hline & Open & 12 & 19 & \\
\hline & Lap converted to open & 3 & 4 & \\
\hline \multirow[t]{3}{*}{ Size of tumor (in cm) (mean) } & Conn's & 1.6 & 1.8 & 0.46 \\
\hline & Cushing's & 4.5 & 3.5 & \\
\hline & PCC & 5.1 & 4.4 & \\
\hline \multirow[t]{3}{*}{ Side of surgery } & Right & 69 & 69 & 0.001 \\
\hline & Left & 40 & 37 & \\
\hline & Bilateral & 6 & 22 & \\
\hline \multirow[t]{3}{*}{ Grade of hypertension (pre-op) } & Grade I & 76 & 20 & 0.001 \\
\hline & Grade II & 23 & 55 & \\
\hline & Grade III & 16 & 53 & \\
\hline
\end{tabular}


Contd...

\begin{tabular}{llccc}
\hline Parameters & & Cohort I cohort (East) $(n=115)$ & Cohort II cohort (West) $(n=128)$ & $p$ value \\
\hline Grade of hypertension (postoperative) & Normal & 93 & 88 & 29 \\
& Grade I & 9 & 10 & 0.51 \\
& Grade II & 13 & 1 & 46 \\
Classes of medications & Grade III & 0 & 53 & 95 \\
& CCB & 96 & 23 & 0.001 \\
& BB & 58 & 29 & 0.001 \\
Cure of hypertension & AB & 44 & 35 & 0.02 \\
All-cause mortality & Diuretics & 35 & 88 (69) & 0.03 \\
\hline
\end{tabular}

$\mathrm{CCB}$, calcium channel blocker; BB, beta-blocker; $\mathrm{AB}$, alpha-blocker; $\mathrm{ACEl}$, acetylcholine esterase inhibitors; PCC, pheochromocytoma

Table 3: The mean preoperative and postoperative systolic and diastolic blood pressures between the two cohorts $(p=0.001)$

\begin{tabular}{|c|c|c|c|c|c|c|c|c|}
\hline & \multicolumn{4}{|c|}{ Cohort I $(n=115)$} & \multicolumn{4}{|c|}{ Cohort II $(n=128)$} \\
\hline & SBPPre & SBP Post & DBP Pre & DBP Post & SBPPre & SBP Post & DBP Pre & DBP Post \\
\hline Conn's & 154.37 & 122.28 & 90.7 & 78.14 & 174.08 & 134.06 & 97.2 & 82.66 \\
\hline PCC & 156.81 & 113.03 & 82.77 & 77.9 & 173.27 & 133.46 & 96.7 & 78.34 \\
\hline Cushing's & 160.79 & 103.76 & 98.72 & 84.02 & 160.64 & 127.00 & 92.00 & 79.64 \\
\hline
\end{tabular}

SBP; systolic blood pressure, DBP; diastolic blood pressure; PCC, pheochromocytoma
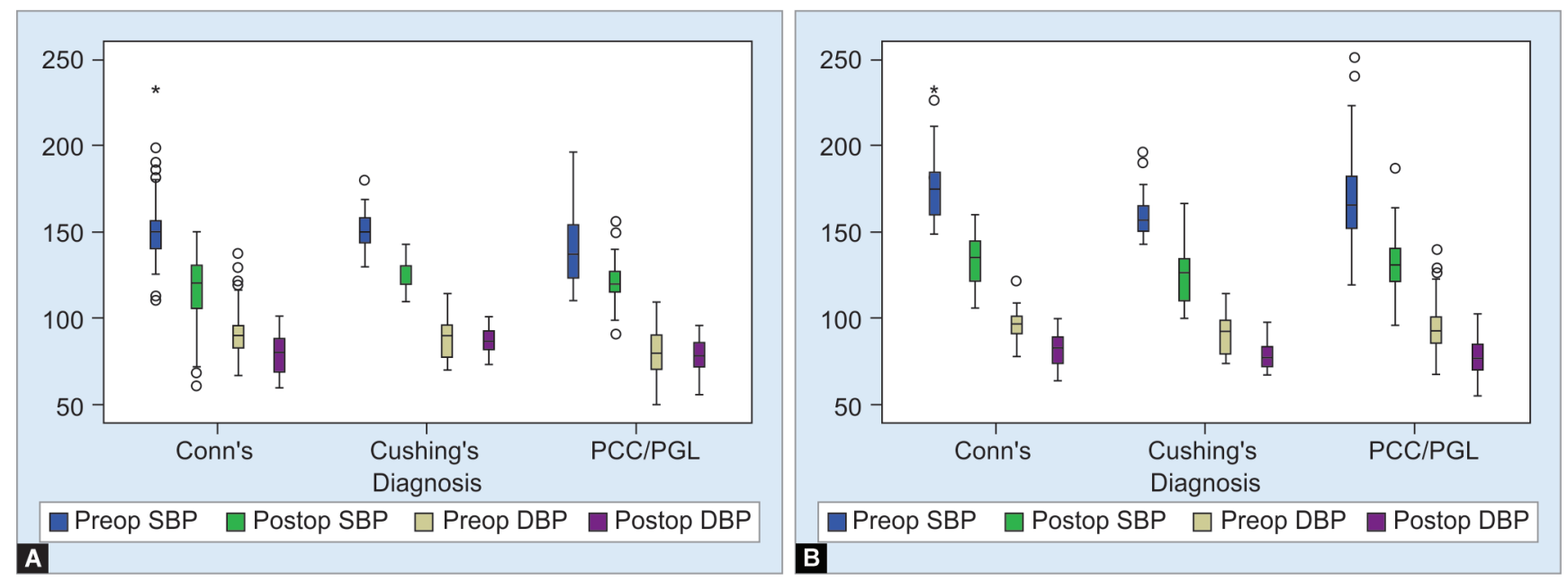

Figs $1 \mathrm{~A}$ and B: The differences in preoperative (preop) and postoperative (postop) systolic and diastolic blood pressures in the two cohorts are based on the etiology

drop of SBP (32 vs 38\%) ( $p=0.07)$ and DBP (10 vs $16 \%)(p=0.06)$ between the two cohorts. The number of patients from the two cohorts who had a reduction in the class of drugs and reduction in standard dose is shown in Figure 2.

Of the 34 patients who were not cured of cohort I, 25 (74\%) had a reduction in the number of classes of drugs and 5 (14\%) had a reduction in the standard dose of the prescribed medications. In cohort II, 34 (85\%) had a reduction in the number of classes of drugs and $5(13 \%)$ had a reduction in the standard dose of the prescribed medications. The association of reduction in a class of antihypertensives and cure between the two cohorts is shown ( $p$ $=0.03$ ). An increase in antihypertensive class was required in 4 (12\%) patients from cohort I and $1(0.7 \%)$ patient from cohort II to maintain hypertension. None of the parameters like age, gender, number of antihypertensive agents and duration were predictors of persistence of $\mathrm{HT}$.

In terms of complications, three patients from cohort I and four patients from cohort II required conversion from laparoscopic to open procedure. The reasons for conversion in cohort I were bleeding in one patient and CBD injury in another due to dense adhesions from previous abdominal surgery requiring a choledochojujenostomy. The third patient sustained a ureteric injury during a posterior retroperitoneal approach due to distorted anatomy from previous laparoscopic anterior resection. In cohort II, conversions to open procedure were for bleeding. All-cause mortality within 30 days of surgery in both the cohorts was similar and was due to cardiovascular accidents (stroke and myocardial infarction). 
Hypertension Cure and Reducing Pill Burden after Adrenalectomy for Endocrine Hypertension of Adrenal Origin

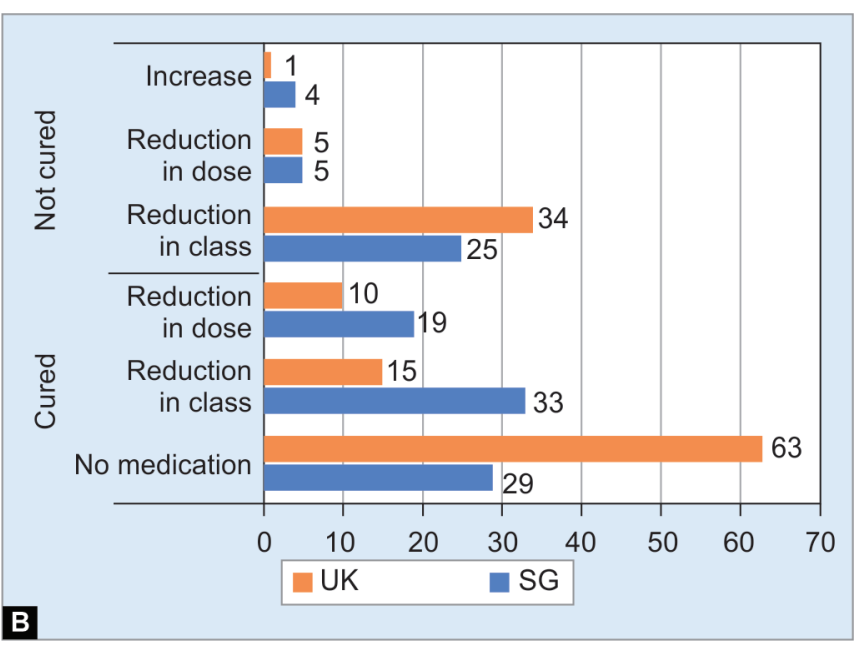

Fig. 2: Outcomes of cure in the two cohorts highlighting a reduction in dose and class in medications

\section{Discussion}

The study is the first comparative study between the East and West in terms of outcomes of endocrine hypertension and the findings are as follows: different etiology of hypertension with a higher rate of Conn's in the East and PCC/PPGL in the west, more left-sided tumors in the East compared to right-sided tumors in the West, grade I and II hypertension in the East compared to grade II and III hypertension in the West, significant differences in the mean pre-op SBP and DBP and choice of antihypertensive medications between the two cohorts. The cure rates, improved blood pressure control, complications from surgery, and overall mortality were similar between the cohorts.

One of the interesting findings of the study is the difference in etiology between the two cohorts in that the predominant cause of endocrine hypertension was Conn's adenoma in Asia and PCC/PPGL in the UK. An earlier study from Singapore showed the prevalence of PA to be around $5 \%$ of adults with hypertension, but the actual figures could be closer to $8 \% .{ }^{18}$ The prevalence of PA increased from 5.6 cases per year in the early ' 90 s to 12.6 per year in the later years due to improved screening. ${ }^{19}$ A similar study from Japan showed the prevalence of endocrine hypertension to be around $9 \%$ with a significant proportion of the cases attributed to PA. ${ }^{20}$

In a cross-sectional study from the UK published recently, the prevalence of PA in newly diagnosed, never treated hypertension was found to be around 3\%; ${ }^{21}$ slightly lower compared to other published studies from Europe with prevalence rates of about $5-8 \%{ }^{22,23}$ There is a difference in healthcare expenditure between the two countries with Singapore currently spending about $4.4 \%$ and the UK about 9.6\% of the GDP on healthcare based on the 2017 statistics published by the World Health Organization, ${ }^{24}$ this does not explain the difference in the higher prevalence of PA in Asia. The differences between the prevalence rates of PA between the two cohorts could be attributed to possibly increased screening in Asia, early referral to specialist clinics for further investigations ${ }^{25}$ and underdiagnosis in UK. ${ }^{26}$

The epidemiological data pertaining to the incidence of PCC/ PPGL are sparse. A nationwide Dutch study showed the incidence of PCC to be rising over the last 2-3 decades, mostly due to improved imaging. ${ }^{27}$ In patients with hypertension, the prevalence of PCC is estimated to be in the West $0.1-0.6 \%,{ }^{28}$ and in Asia prevalence is low, around 2 per 100,000 population. ${ }^{29}$ The study from the US using the Health Care Utilization Project Nationwide Inpatient Sample (HCUP-NIS) for a period of 5 years showed the incidence of hormone active tumors including PCC to be significantly higher in Caucasians in comparison to Asians and Blacks. ${ }^{30}$ Over $70 \%$ of the patients presented with hypertension in the series.

The National Audit data published by the British Association of Endocrine and Thyroid Surgeons in 2017 showed that over 30\% of the adrenalectomy performed was for PCC compared to $18 \%$ for Conn's and Cushing's adenoma. ${ }^{31}$ The results presented in our study show a similar pattern with the UK cohort consisting of Caucasians only showing a higher incidence of PCC/PPGL compared to the Asian cohort. The incidence of germline mutations such as VHL, MEN 2, and NF-1 associated with PCC is low in Asia due to lack of acceptance for genetic screening by the patients ${ }^{32,33}$ and non-referral to genetic services. ${ }^{34}$ In our study, there were only 6 patients with germline mutations (MEN2a and MEN2b) from the Asian cohort in comparison to 22 germline mutations (MEN2a, MEN2b, SDH, VHL, and NF) in the Western cohort.

There was a difference in the laterality of the tumors between the two cohorts with right-sided lesions seen more in the UK compared to the left-sided lesions in Asia. Looking at individual pathologies, Conn's adenoma was predominantly seen on left in Asia, with no laterality in the other two pathologies. Many studies pertaining to adrenalectomy for Conn's syndrome showed a predominance of left-sided lesions. ${ }^{6,35-37}$ In the UK, PCC/PPGL and Cushing's adenoma was more predominantly seen on the right. The differences in laterality are more likely due to selection bias.

The overall preoperative BP appeared to be lower in Singapore when compared to the UK and this may be due to the more stringent screening for secondary hypertension and earlier detection of secondary causes of hypertension. Evidence suggests that PA increases with the grade of hypertension, with higher grades seen in resistant hypertension. Similarly, high grades of BP are seen in PCC, with over $60 \%$ presenting with sustained hypertension and $30 \%$ with a paroxysmal disease, and about a third presenting with hypertensive crisis. Differences seen in the grade of hypertension and the mean pre- and postoperative BP (systolic and diastolic) between the two cohorts are more due to the underlying predominant pathology in each. The above also explains the difference in the choice of antihypertensive medications in the two cohorts prescribed for the underlying conditions.

Despite the differences in the etiology of hypertension in the two cohorts, the cure rates were similar and are consistent with published series. ${ }^{35,38-42}$ A significant proportion of patients had normalization of BP through the reduction in the number of antihypertensive agents or in the standard dosages of the medications. Studies have shown that risk factors associated with persistent hypertension include advanced age ${ }^{43,44}$ duration of hypertension, ${ }^{41,44,45}$ male gender, ${ }^{45}$ and family history of hypertension. ${ }^{46,47}$ There were no significant predictors of the persistence of HT in this study.

The reasons for persistent hypertension despite the removal of the trigger could be the coexistence of primary hypertension and the damage inflicted to the vascular endothelium and the kidneys. ${ }^{48}$ Recently, gene expression studies on patients with persistent hypertension following adrenalectomy showed a few genes associated with biological pathways related to hypertension to be over- or under-expressed. ${ }^{42}$ Response of endocrine hypertension 
to adrenalectomy therefore can be heterogeneous similar to that reported in this study. Rossi et al. showed that vascular mediato-lumen ratio and media width were greater in patients with persistent hypertension due to the breakdown in some of the protective reflex mechanisms to control the blood pressure. ${ }^{49}$

To summarize, there is a difference in the incidence of adrenal conditions leading to hypertension between the East and the West. The outcomes of intervention in the form of hypertensive cure and complications are excellent in expert centers irrespective of the underlying adrenal cause. However, a small proportion of patients will not have their hypertension cured due to vascular remodeling and coexistent essential hypertension. Perhaps early diagnosis and treatment will mitigate the end-organ damage and vascular endothelial changes associated with long-standing endocrine hypertension.

The paper was accepted as a poster presentation for the European Association of Endocrine Surgeons 2020 Meeting in Greece.

\section{References}

1. Kannel WB. Blood pressure as a cardiovascular risk factor: prevention and treatment. JAMA 1996;275(20):1571-1576. DOI: 10.1001/ jama.1996.03530440051036.

2. Quinkler M, Lepenies J, Diederich S. Primary hyperaldosteronism. Exp Clin Endocrinol Diabetes 2002;110(6):263-271. DOI: 10.1055/s2002-34588.

3. Camelli S, Bobrie G, Postel-Vinay N, et al. LB01.11: Prevalence of secondary hypertension in young hypertensive adults. J Hypertens 2015;33(Suppl 1):e47. DOI: 10.1097/01.hjh.0000467473.21331.70.

4. Funder JW. Primary aldosteronism as a public health issue. Lancet Diabetes Endocrinol 2016;4(12):972-973. DOI: 10.1016/s22138587(16)30272-8.

5. Liao $\mathrm{CH}$, Wu V, Jeff Chueh $\mathrm{S}$, et al. Outcome of surgery for primary hyperaldosteronism. World J Surg 2012(5):Online ahead of print 10.1007/s00268-011-1370-6.

6. Waldmann J, Maurer L, Holler J, et al. Outcome of surgery for primary hyperaldosteronism. World J Surg 2011;35(11):2422-2427. DOI: 10.1007/s00268-011-1221-5.

7. Martucci VL, Pacak K. Pheochromocytoma and paraganglioma: diagnosis, genetics, management, and treatment. Curr Probl Cancer 2014;38(1):7-41. DOI: 10.1016/j.currproblcancer.2014.01.001.

8. Nieman LK, Biller BM, Findling JW, et al. Treatment of Cushing's syndrome: an endocrine society clinical practice guideline. J Clin Endocrinol Metab 2015;100(8):2807-2831. DOI: 10.1210/jc.2015-1818.

9. Gouli A, Kaltsas G, Tzonou A, et al. High prevalence of autonomous aldosterone secretion among patients with essential hypertension. Eur J Clin Investigat 2011;41(11):1227-1236. DOI: 10.1111/j.13652362.2011.02531.x.

10. Calhoun DA, Nishizaka MK, Zaman MA, et al. Hyperaldosteronism among black and white subjects with resistant hypertension. Hypertension 2002;40(6):892-896. DOI: 10.1161/01. hyp.0000040261.30455.b6.

11. Baid S, Nieman LK. Glucocorticoid excess and hypertension. Curr Hypertens Rep 2004;6(6):493-499. DOI: 10.1007/s11906-004-0046-0.

12. Magiakou MA, Mastorakos G, Oldfield EH, et al. Cushing's syndrome in children and adolescents--presentation, diagnosis, and therapy. N Eng J Med 1994;331(10):629-636. DOI: 10.1056/ NEJM199409083311002.

13. Stewart PM, Walker BR, Holder G, et al. 11 beta-hydroxysteroid dehydrogenase activity in Cushing's syndrome: explaining the mineralocorticoid excess state of the ectopic adrenocorticotropin syndrome. J Clin Endocrinol Metabol 1995;80(12):3617-3620. DOI: 10.1210/jcem.80.12.8530609.

14. Bravo EL, Tagle R. Pheochromocytoma: state-of-the-art and future prospects. Endoc Rev 2003;24(4):539-553. DOI: 10.1210/er.2002-0013.
15. Sharma ST, Nieman LK. Cushing's syndrome: all variants, detection, and treatment. Endocrinol Metabol Clin 2011;40(2):379-391. DOI: 10.1016/j.ecl.2011.01.006.

16. Zhou Y, Zhang M, Ke S, et al. Hypertension outcomes of adrenalectomy in patients with primary aldosteronism: a systematic review and meta-analysis. BMC Endocri Disord 2017;17(1):61-69. DOI: 10.1186/ s12902-017-0209-z.

17. Williams B, Mancia G, Spiering W, et al. 2018 ESC/ESH Guidelines for the management of arterial hypertension: the task force for the management of arterial hypertension of the European Society of Cardiology (ESC) and the European Society of Hypertension (ESH). Eur Heart J 2018;39(33):3021-3104. DOI: 10.1093/eurheartj/ ehy339.

18. Loh K-C, Koay ES, Khaw M-C, et al. Prevalence of primary aldosteronism among Asian hypertensive patients in Singapore1.J Clin Endocrinol Metabol 2000;85(8):2854-2859. DOI: 10.1210/jcem.85.8. 6752.

19. Mulatero P, Stowasser M, Loh K-C, et al. Increased diagnosis of primary aldosteronism, including surgically correctable forms, in centers from five continents. J Clin Endocrinol Metabol 2004;89(3):1045-1050. DOI: 10.1210/jc.2003-031337.

20. Omura M, Saito J, Yamaguchi K, et al. Prospective study on the prevalence of secondary hypertension among hypertensive patients visiting a general outpatient clinic in Japan. Hypert Res 2004;27(3):193-202. DOI: 10.1291/hypres.27.193.

21. Käyser SC, Deinum J, de Grauw WJ, et al. Prevalence of primary aldosteronism in primary care: a cross-sectional study. Br J Gen Pract 2018;68(667):e114-e122. DOI: 10.3399/bjgp18X694589.

22. Fogari R, Preti P, Zoppi A, et al. Prevalence of primary aldosteronism among unselected hypertensive patients: a prospective study based on the use of an aldosterone/renin ratio above 25 as a screening test. Hypertens Res 2007;30(2):111-117. DOI: 10.1291/hypres.30.111.

23. Westerdahl C, Bergenfelz A, Isaksson A, et al. Primary aldosteronism among newly diagnosed and untreated hypertensive patients in a Swedish primary care area. Scand J Prim Health Care 2011;29(1):57-62. DOI: 10.3109/02813432.2011.554015.

24. WHO. Global Health Expenditure Database. WHO; 2017.https://apps. who.int/nha/database/Home/Index/en.

25. Puar T, Mok $Y$, Debajyoti $R$, et al. Secondary hypertension in adults. Singapore Med J 2016;57(5):228-232. DOI: 10.11622/smedj. 2016087.

26. Arlt W. A detour guide to the endocrine society clinical practice guideline on case detection, diagnosis and treatment of patients with primary aldosteronism. Eur J Endocrinol 2010;162(3):435-438. DOI: 10.1530/eje-09-0869.

27. Berends AMA, Buitenwerf E, de Krijger RR, et al. Incidence of pheochromocytoma and sympathetic paraganglioma in the Netherlands: a nationwide study and systematic review. Eur J Intern Med 2018;51:68-73. DOI: 10.1016/j.ejim.2018.01.015.

28. Lenders JW, Eisenhofer G, Mannelli M, et al. Phaeochromocytoma. Lancet 2005;366(9486):665-675. DOI: 10.1016/S0140-6736(05) 67139-5.

29. Kim JH, Moon H, Noh J, et al. Epidemiology and prognosis of pheochromocytoma/paraganglioma in Korea: a nationwide study based on the national health insurance service. Endocri Metab (Seoul) 2020;35(1):157-164. DOI: 10.3803/enm.2020.35.1.157.

30. Parikh PP, Rubio GA, Farra JC, et al. Nationwide review of hormonally active adrenal tumors highlights high morbidity in pheochromocytoma. J Surg Res 2017;215:204-210. DOI: 10.1016/j. jss.2017.04.011.

31. Chadwick D. The British Association of Endocrine and Thyroid Surgeons. Fifth National Audit Report 2017. April ed., Oxford: Dendrite Clinical Systems Ltd; 2017. pp. 150-154.

32. Sriphrapradang C, Choopun K, Tunteeratum A, et al. Genotypephenotype correlation in patients with germline mutations of $\mathrm{VHL}$, RET, SDHB, and SDHD Genes: Thai experience. Clin Med Insights Endocrinol Diabetes 2017;10:1179551417705122-1179551417705122. DOI: $10.1177 / 1179551417705122$ 
33. Khadilkar K, Sarathi V, Kasaliwal R, et al. Predictors of malignancy in patients with pheochromocytomas/paragangliomas: Asian Indian experience. Endocr Connect 2016;5(6):89-97. DOI: 10.1530/EC-160086.

34. Chew WHW, Courtney E, Lim KH, et al. Clinical management of pheochromocytoma and paraganglioma in Singapore: missed opportunities for genetic testing. Molecul Genet Geno Med 2017;5(5):602-607. DOI: 10.1002/mgg3.313.

35. Pang TC, Bambach C, Monaghan JC, et al. Outcomes of laparoscopic adrenalectomy for hyperladosteronism. ANZ J Surg 2007;77(9):768773. DOI: 10.1111/j.1445-2197.2007.04225.x.

36. Worth PJ, Kunio NR, Siegfried I, et al. Characteristics predicting clinical improvement and cure following laparoscopic adrenalectomy for primary aldosteronism in a large cohort. Am J Surg 2015;210(4):702709. DOI: 10.1016/j.amjsurg.2015.05.033.

37. Trésallet C, Salepçioglu H, Godiris-Petit G, et al. Clinical outcome after laparoscopic adrenalectomy for primary hyperaldosteronism: the role of pathology. Surgery 2010;148(1):129-134. DOI: 10.1016/j. surg.2009.11.020.

38. Hannon M, Sze W, Carpenter R, et al. Clinical outcomes following unilateral adrenalectomy in patients with primary aldosteronism. QJM 2017;110(5):277. DOI: 10.1093/qjmed/hcw194.

39. Burrello J, Burrello A, Stowasser M, et al. The primary aldosteronism surgical outcome score for the prediction of clinical outcomes after adrenalectomy for unilateral primary aldosteronism. Ann Surg 2020;272(6):1125-1132. DOI: 10.1097/SLA.0000000000003200.

40. Katabami T, Fukuda H, Tsukiyama $H$, et al. Clinical and biochemical outcomes after adrenalectomy and medical treatment in patients with unilateral primary aldosteronism. J Hypertens 2019;37(7):15131520. DOI: $10.1097 / \mathrm{HJH} .0000000000002070$.

41. Brunt LM, Moley JF, Doherty GM, et al. Outcomes analysis in patients undergoing laparoscopic adrenalectomy for hormonally active adrenal tumors. Surgery 2001;130(4):629-635. DOI: 10.1067/ msy.2001.116920.

42. Xie L-F, Ouyang J-Z, Wang A-P, et al. Gene expression profile of persistent postoperative hypertension patients with aldosteroneproducing adenomas. Chin Med J (Engl) 2015;128(12):1618-1626.

43. Sapienza $P$, Cavallaro A. Persistent hypertension after removal of adrenal tumours. Eur J Surg 1999;165(3):187-192. DOI: 10.1080/110241599750007027.

44. Lumachi F, Ermani M, Basso SMM, et al. Long-term results of adrenalectomy in patients with aldosterone-producing adenomas: multivariate analysis of factors affecting unresolved hypertension and review of the literature. Am Surg 2005;71(10):864. DOI: 10.1177/000313480507101015.

45. Citton M, Viel G, Rossi GP, et al. Outcome of surgical treatment of primary aldosteronism. Langenbeck's Archi Surg 2015;400(3):325331. DOI: 10.1007/s00423-014-1269-4.

46. Sawka AM, Young WF, Thompson GB, et al. Primary aldosteronism: factors associated with normalization of blood pressure after surgery. Ann Int Med 2001;135(4):258. DOI: 10.7326/0003-4819-1354-200108210-00010.

47. Proye CAG, Mulliez EAR, Carnaille BML, et al. Essential hypertension: first reason for persistent hypertension after unilateral adrenalectomy for primary aldosteronism? Surgery 1998;124(6):1128-1133. DOI: 10.1067/msy.1998.93108.

48. Wolf-Maier K, Cooper RS, Kramer H, et al. Hypertension treatment and control in five European countries, Canada, and the United States. Hypertension 2004;43(1):10-17. DOI: 10.1161/01. Hyp.0000103630.72812.10.

49. Rossi GP, Bolognesi M, Rizzoni D, et al. Vascular remodeling and duration of hypertension predict outcome of adrenalectomy in primary aldosteronism patients. Hypertension 2008;51(5):1366-1371. DOI: 10.1161/hypertensionaha.108.111369. 